Пути оптимизации

дистанционной

лучевой терапии рака

предстательной железы

\title{
Балканов А.C.
}

Балканов Андрей Сергеевич - д-р мед. наук, заведующий радиологическим отделением'

$\triangle 129110$, г. Москва, ул. Щепкина, 61/2-7, Российская Федерация.

Тел.: +7 (495) 6811907.

E-mail: andreybalkanov@yandex.ru
${ }^{1}$ ГБУз МО «Московский областной научно-исследовательский клинический институт им. М.Ф. Владимирского»; 129110, г. Москва, ул. Щепкина, 61/2, Российская Федерация
Актуальность. Основанием для применения дистанционной гамма-терапии (ДГТ) у пациентов с раком предстательной железы послужили данные об ее эффективности в сочетании с современными планирующими устройствами и более низкая себестоимость по сравнению с использованием линейного медицинского ускорителя.

Цель - оценить влияние применения ДГТ у пациентов с раком предстательной железы с использованием компьютерной программы 3D-планирования Амфора на показатель 5-летней выживаемости в зависимости от основных прогностических критериев эффективности лечения.

Материал и методы. В исследование включены 34 пациента с раком предстательной железы, которые получили низкодозную ДГТ в суммарной очаговой дозе 62-70 Гр. Андрогенная депривация имела место у 73,5\% пациентов в течение 6-12 месяцев.

Результаты. У пациентов с исходным уровнем простатспецифического антигена в крови <20 нг/мл показатель 5-летней выживае- мости оказался наивысшим и составил 86\%. Установлено, что в условиях сочетанного применения с андрогенной депривацией суммарная очаговая доза при низкодозной ДГТ (<64 Гр; $\geq 64$ Гр) не оказывает статистически значимого влияния на показатель 5-летней выживаемости $(p=0,61)$.

Заключение. Полученные нами результаты лишь первый шаг на пути установления критериев, позволяющих выбрать способ дистанционной лучевой терапии при раке предстательной железы. Оптимизация определения тактики лечения на основании тщательного анализа исходных данных, в том числе о степени тяжести сопутствующей патологии, сделает использование Дгт более обоснованным, что будет способствовать снижению себестоимости лечения.

Ключевые слова: рак предстательной железы, лучевая терапия, 5-летняя выживаемость, себестоимость лучевой терапии, простатспецифический антиген, старческий возраст, сопутствующая патология.

doi: 10.18786/2072-0505-2015-41-46-51 
Д истанционную гамма-терапию (ДГТ) стали широко применять в онкологии с середины прошлого столетия. В 1951 г. в Канаде была введена в строй первая установка для ДГТ Eldorado А, принцип действия которой основывался на способности изотопа кобальта-60 излучать гамма-кванты. Уже в 1953 г. в США было налажено серийное производство таких установок. В 1968 г. компания Varian paзработала первый компактный медицинский ускоритель Clinac 6, и к концу прошлого века при проведении дистанционной лучевой терапии линейные медицинские ускорители повсеместно вытеснили установки для ДГТ. Основным преимуществом линейных медицинских ускорителей считается возможность за счет высокой энергии пучка фотонов и использования устройств, позволяющих более точно планировать и осуществлять дистанционную лучевую терапию, увеличить суммарную дозу облучения (СОД), не опасаясь роста частоты лучевых осложнений (например, при раке предстательной железы (РПЖ) с 70 до 80-85 Гр), что и обеспечивает высокие показатели общей выживаемости [1]. Среди таких устройств следует прежде всего отметить многолепестковый коллиматор (англ. multileaf collimator) и компьютерные программы, позволяющие объемное (3D) планирование дистанционной лучевой терапии по данным исследования самой опухоли и окружающих ее тканей методом рентгеновской компьютерной томографии. Такая высокодозная дистанционная лучевая терапия получила название конформной (кДЛТ).

В последнее время снова стали появляться публикации о возможности эффективного применения ДГТ в сочетании с современными планирующими устройствами у пациентов с РПЖ [2]. Основанием для подобного рода исследований послужила более низкая стоимость ДГТ по сравнению с использованием линейных медицинских ускорителей. В этой ситуации возникает необходимость в выработке четких критериев применения каждого из вышеобозначенных видов дистанционной лучевой терапии в этой группе онкологических больных. Такой подход представляется еще более актуальным, если учесть, что очень часто в сочетании с дистанционной лучевой терапией при РПЖ используется андрогенная депривация, способная даже при применении в виде монотерапии обеспечить высокую продолжительность жизни. Разработка эффективного сочетания существующих способов дистанционной лучевой терапии и андрогенной депривации, а также использование в качестве критериев прогноза дистанционной лучевой терапии показателей, объективно характеризующих особенности течения РПЖ у каждого конкретного пациента, по нашему мнению, могут способствовать оптимизации дистанционной лучевой терапии.

Нами предпринят сравнительный анализ 5-летней выживаемости пациентов с РПЖ, которым проводилась ДГТ с использованием компьютерной программы 3D-планирования Амфора, в зависимости от некоторых основных критериев прогноза лечения РПЖ.

\section{Материал и методы}

С 2007 по 2009 г. у 38 пациентов с диагнозом РПЖ стадии Т2-3, N0-1 использовалась ДГТ в режиме классического фракционирования: разовая очаговая доза - 2 Гр, СОД - 62-70 Гр. В связи с отсутствием данных о продолжительности жизни 4 пациентов они были исключены из исследования. В результате сравнительный анализ проведен у 34 пациентов. Данные, характеризующие некоторые показатели состояния пациентов на момент начала ДГТ, представлены в таблице.

При планировании ДГТ в клинический объем мишени (Clinical Target Volume - CTV) включались вся предстательная железа и семенные пузырьки. Планируемый объем облучения (Planning Target Volume - PTV) определялся как $\mathrm{CTV}+1$ см. Зона расположения подвздошных лимфатических узлов включалась в $60-80 \%$ изодозную кривую. Для планирования ДГТ использовались симулятор с томографической приставкой Акьюити фирмы «Вариан» в сочетании с компьютерной программой 3D-планирования Амфора. ДГТ осуществлялась на гамма-терапевтических установках Терабалт (Чехия) и Рокус (Россия). Коллиматор на установке Терабалт позволял создавать асимметрию поля облучения. Если исходный уровень простатспецифического антигена (ПСА) крови превышал 10 нг/мл, назначали андрогенную депривацию: использовались аналоги гонадотропин-рилизинг гормона (гозерелин, препарат Золадекс), антиандрогены (бикалутамид, препарат Касодекс) или максимальная андрогенная блокада. Длительность андрогенной депривации составляла от 6 до 12 месяцев.

Рецидив диагностировали либо в случае двукратного увеличения уровня ПСА крови более 2 нг/мл выше наименьшего значения этого показателя, зафиксированного после завершения ДГТ и/или андрогенной депривации, либо при инструментальной визуализации метастаза.

Эффективность применения ДГТ у пациентов с РПЖ оценивали с использованием показателя 5-летней выживаемости, вычисленного 


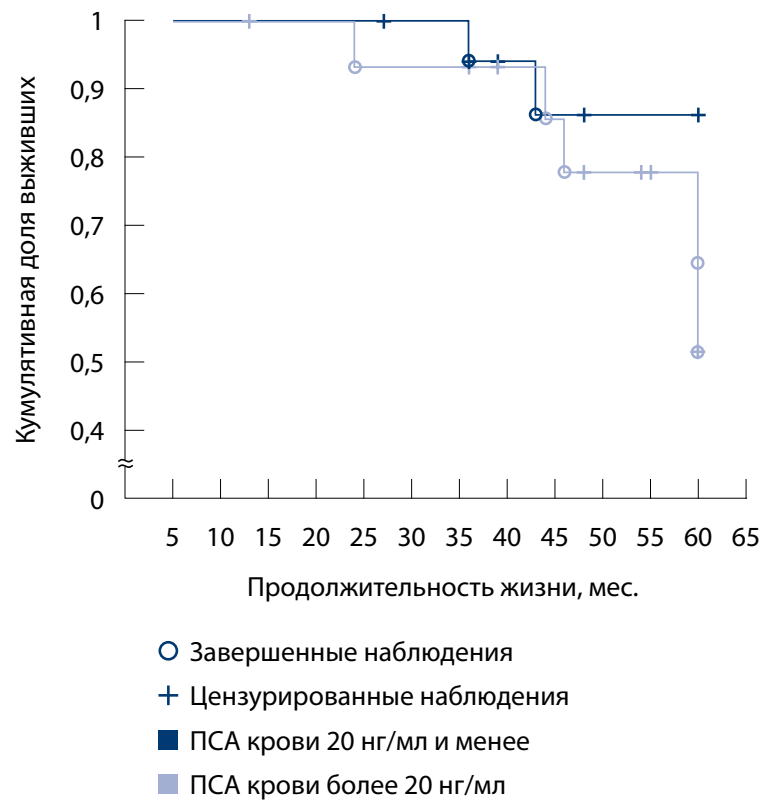

Рис. 1. Пятилетняя выживаемость пациентов с РПЖ после проведения ДГТ в зависимости от исходного уровня простатспецифического антигена (ПСА) крови (оценка методом Каплана - Мейера)

по методике Каплана - Мейера. Сравнительный анализ этого показателя проводили в группах, разделенных по следующим признакам: уровень ПСА крови (<20 нг/мл, $\geq 20$ нг/мл), применение андрогенной депривации, СОД при ДГТ (<64 Гр, $\geq 64$ Гр).

В ходе статистического анализа использовалась программа Statistica 6.1 for Windows (StatSoft Inc., США). Разница показателей считалась достоверной при $\mathrm{p}<0,05$.

\section{Результаты}

Сравнительный анализ 5-летней выживаемости среди пациентов с РПЖ, которым был проведен курс ДГТ, в зависимости от исходного уровня ПСА крови осуществлен в двух группах: 1-ю группу составили 17 пациентов, исходный ПСА крови у которых определялся на уровне менее 20 нг/мл, 2-ю группу - еще 17 пациентов с исходным уровнем ПСА крови, равным или превышающим 20 нг/мл. В 1-й группе 5-летняя выживаемость оказалась выше, чем во 2-й: 86 и 52\% соответственно (рис. 1), однако разница была статистически не значимой $(\mathrm{p}=0,22)$.

На следующем этапе выполнен сравнительный анализ эффективности ДГТ в зависимости от использования андрогенной депривации. Показатель 5-летней выживаемости

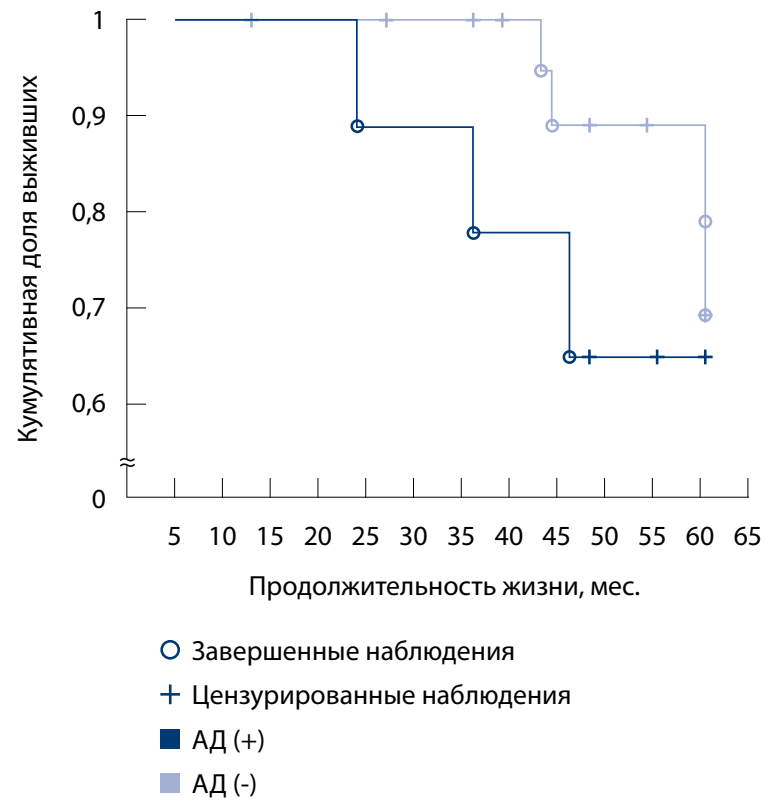

Рис. 2. Пятилетняя выживаемость пациентов с РПЖ после проведения ДГТ в зависимости от применения у них андрогенной депривации (оценка методом Каплана - Мейера);

АД (+) - пациенты получали андрогенную депривацию,

АД (-) - пациенты не получали лечение андрогенной депривацией

у 25 пациентов с РПЖ, которые в сочетании с ДГТ получали лечение андрогенной депривацией, равнялся 65\% и статистически значимо не отличался от аналогичного показателя среди 9 пациентов, андрогенная депривация у которых не применялась, - 69\% (р=0,3) (рис. 2).

На заключительном этапе исследования выполнен сравнительный анализ эффективности лечения пациентов с РПЖ в зависимости от СОД при проведении ДГТ (рис. 3). Установлено, что 5-летняя выживаемость пациентов, у которых ДГТ была завершена на СОД менее 64 Гр, была выше по сравнению с таковой у пациентов, получавших СОД 64 Гр и более: 77 и 58\% соответственно, при этом межгрупповые различия не достигли уровня статистической значимости $(\mathrm{p}=0,61)$.

\section{Обсуждение}

Дистанционная лучевая терапия у пациентов с РПЖ стала широко применяться с середины 50-х гг. прошлого века в виде ДГТ до СОД 60-65 Гр. Уже первые результаты, полученные в реальной клинической практике, показали высокую эффективность этого способа лечения: 5-летняя выживаемость увеличилась до 70\% [3]. Высокая частота местного рецидива после ДГТ (20\%) наблюдалась только при местнораспространенной форме РПЖ $[4,5]$. В начале 1980 -х стало очевидно, что резкое 


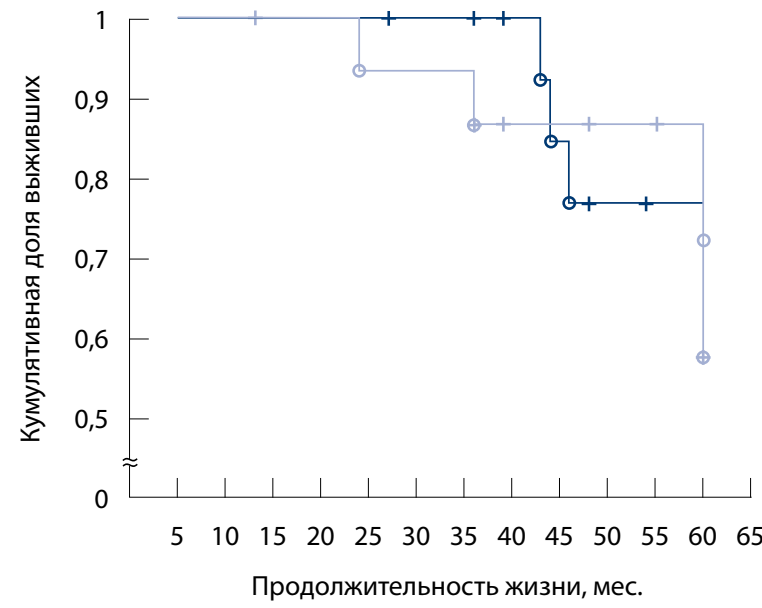

О Завершенные наблюдения

+ Цензурированные наблюдения

СОД менее 64 Гр

СОД 64 Гр и выше

Рис. 3. Пятилетняя выживаемость пациентов с РПЖ в зависимости от суммарной очаговой дозы (СОД) при проведении ДГТ (оценка методом Каплана - Мейера)

снижение вероятности местного рецидива возможно только при увеличении СОД до 70 Гр и выше [5]. Практически это удалось реализовать в 1990-х гг. после внедрения более совершенных принципов планирования и линейных медицинских ускорителей, то есть при помощи кДЛТ. Увеличение СОД в зоне РПЖ обеспечило рост 5-летней выживаемости до 84,9\% [1]. В последние годы появляется все больше публикаций о применении у пациентов с РПЖ более сложных методик кДЛТ (например, с модуляцией интенсивности; Intensity Modulated Radiotherapy - IMRT) и радиохирургического способа облучения, что обеспечивает 5-летнюю безрецидивную выживаемость на уровне более $90 \%$ [6].

Совершенствование способов дистанционной лучевой терапии, применяемых при РПЖ, привело к росту стоимости лечения. Так, по данным зарубежных источников, в 70-80 гг. прошлого столетия стоимость дистанционной лучевой терапии при РПЖ составляла 6,75 тыс. долларов США [7]. Через 10 лет, по мере оснащения лечебных учреждений оборудованием для проведения кДЛТ, стоимость лечения возросла до 13,8 тыс. долларов США [8]. Использование новейшей методики кДЛТ - IMRT - у пациентов с РПЖ увеличило плату за лечение до 18 тыс. долларов США. Применение протонотерапии обходится еще дороже - в сумму около 32 тыс. долларов США [9]. Таким образом, увеличение 5-летней
Клинико-демографическая характеристика пациентов с РПЖ, включенных в исследование $(n=34)$

\begin{tabular}{|c|c|}
\hline Показатель & $\begin{array}{l}\text { Количество пациентов, } \\
\text { n (\%) }\end{array}$ \\
\hline \multicolumn{2}{|l|}{ Возраст } \\
\hline средний (менее 60 лет) & $8(23,5)$ \\
\hline пожилой (60-69 лет) & $9(26,5)$ \\
\hline старческий (70 лет и более) & $17(50)$ \\
\hline \multicolumn{2}{|l|}{ Т опухоли } \\
\hline $\mathrm{T} 2$ & $10(29,4)$ \\
\hline T3 & $23(67,6)$ \\
\hline T4 & $1(3)$ \\
\hline \multicolumn{2}{|l|}{ N опухоли } \\
\hline$N(0)$ & $30(88,2)$ \\
\hline$N(1)$ & $4(11,8)$ \\
\hline \multicolumn{2}{|l|}{ Исходный уровень ПСА } \\
\hline$<20$ нг/мл & $17(50)$ \\
\hline$\geq 20$ нг/мл & $17(50)$ \\
\hline \multicolumn{2}{|c|}{$\begin{array}{l}\text { Дифференцировка аденокарциномы } \\
\text { (по Глисону) }\end{array}$} \\
\hline$<7$ баллов & $7(20,6)$ \\
\hline 7 баллов & $17(50)$ \\
\hline >7 баллов & $10(29,4)$ \\
\hline АД (+) & $25(73,5)$ \\
\hline АД (-) & $9(26,5)$ \\
\hline СоД < 64 Гр & $17(50)$ \\
\hline СОД $\geq 64$ Гр & $17(50)$ \\
\hline
\end{tabular}

ПСА - простатспецифический антиген, АД (+) - пациенты получали андрогенную депривацию, АД (-) - пациенты не получали лечение андрогенной депривацией, СОД - суммарная очаговая доза

выживаемости при использовании у пациентов с РПЖ кДЛТ сопровождается двукратным ростом стоимости лечения по сравнению с ДГТ.

В этой связи возникает вопрос: если применение более дешевой, низкодозной ДГТ (в СОД 65-70 Гр) способно в ряде случаев обеспечить уровень, например, 5-летней выживаемости, сравнимый с таковым при использовании высокодозной кДЛТ, насколько целесообразно применение более дорогого способа дистанционной лучевой терапии? Результаты нашего исследования подтверждают необходимость переосмысления роли ДГТ при выборе тактики лечения у пациентов с РПЖ. Так, мы установили, что исходный уровень ПСА крови не оказывал статистически значимого влияния на результат лечения. При этом применение в нашем исследовании современного способа 3D-планирования при проведении ДГТ в СОД 62-70 Гр у пациентов с низким исходным уровнем ПСА крови (<20 нг/мл) обеспечивало уровень 5-летней выживаемости в 86\%. Эти результаты сопоставимы с данными зарубежных авторов, 
полученными при лечении аналогичных групп пациентов с использованием кДЛТ: 5-летняя выживаемость составила 88-89\% [10]. На наш взгляд, это дает основания говорить о равной эффективности двух видов дистанционной лучевой терапии; следовательно, у некоторых пациентов с низким исходным уровнем ПСА крови ДГТ можно использовать как основной способ лечения РПЖ.

По данным ряда авторов, проведение лечения пациентов с РПЖ в старческом возрасте увеличивает 5-летнюю выживаемость лишь на 6\%. Причинами столь незначительного влияния самых современных способов лечения, включая кДЛТ, на выживаемость считаются высокая смертность от сопутствующей патологии, достигающая в этой возрастной категории $80 \%$, и существенное ухудшение качества жизни в первые 5 лет после проведения лечения $[11,12]$.

Суммируя приведенные выше данные, можно сделать вывод о том, что пациенты с РПЖ в старческом возрасте, у которых диагностируется низкий исходный уровень ПСА крови, являются при определенных условиях кандидатами для применения у них ДГТ в сочетании с современными системами 3D-планирования. Ряд исследователей так же, как и мы, получили доказательства того, что проведение ДГТ с использованием современных систем планирования гарантирует дозное распределение в зоне предстательной железы, сравнимое с таковым при применении линейных медицинских ускорителей, а значит, может считаться эффективным способом лечения РПЖ [2, 13]. Таким образом, необходимо проводить тщательный анализ индивидуальных показателей, характеризующих течение РПЖ у каждого конкретного пациента. Результаты такого анализа должны лечь в основу оптимизации дистанционной лучевой терапии у таких пациентов.

Отсутствие в нашем исследовании статистически значимых различий в показателях 5-летней выживаемости в зависимости от СОД при проведении ДГТ представляется нам важным результатом. Похожие данные получены другими авторами: при использовании дистанционной лучевой терапии у пациентов с РПЖ в СОД, равной
68 и 78 Гр, 5-летняя выживаемость не зависела от СОД и составила 82 и $83 \%$ соответственно [14]. Считается, что в ряде случаев у пациентов с РПЖ отсутствие влияния увеличения СОД на выживаемость объясняется андрогенной депривацией, проводимой в сочетании с облучением. Так, установлено, что снижение 5-летней смертности у пациентов с РПЖ возможно только на фоне низкодозной дистанционной лучевой терапии (<70 Гр), но в сочетании с длительным курсом андрогенной депривации, и не наблюдается при применении высокодозной (>70 Гр) кДЛТ в качестве монотерапии [15]. В нашем исследовании большинство $(73,5 \%)$ пациентов получали андрогенную депривацию, что, скорее всего, и предопределило отсутствие достоверного влияния СОД на показатели выживаемости. Стоит отметить, что при назначении андрогенной депривации пациентам, у которых планируется проведение дистанционной лучевой терапии, обязательно следует учитывать характер сопутствующей патологии. Увеличение 5-летней безрецидивной выживаемости на 11\% при сочетанном применении андрогенной депривации и дистанционной лучевой терапии имеет место только у пациентов без тяжелой сопутствующей патологии [16]. Другими словами, разумное сочетанное использование дистанционной лучевой терапии и андрогенной депривации, в том числе низкодозной ДГТ, у пациентов с тяжелой сопутствующей патологией может обеспечить высокий уровень выживаемости при РПЖ при условии резкого снижения себестоимости лечения.

Тем не менее было бы неправомерно делать однозначный вывод, что решение вопроса о выборе способа дистанционной лучевой терапии у пациентов с РПЖ найдено. Наши данные - лишь первый шаг в попытке установить критерии определения метода дистанционной лучевой терапии у таких пациентов при условии соблюдения гарантии высокого качества лечения, с одной стороны, и разумной себестоимости - с другой. Однако мы уверены: продолжение исследований в данном направлении позволит со временем оптимизировать тактику лечения пациентов с РПЖ, обосновав применение ДГТ у конкретного контингента больных. (๕)

\section{Литература / References}

1. Surapaneni A, Schwartz D, Nwokedi E, Rineer J, Rotman M, Schreiber D. Radiation therapy for clinically localized prostate cancer: long-term results of 469 patients from a single institution in the era of dose escalation. J Cancer Res Ther. 2014;10(4):951-6. doi: 10.4103/09731482.138096 .
2. Joshi CP, Dhanesar S, Darko J, Kerr A, Vidyasagar PB, Schreiner LJ. Practical and clinical considerations in Cobalt-60 tomotherapy. J Med Phys. 2009;34(3):137-40. doi: 10.4103/09716203.54847.

3. Bagshaw MA, Ray GR, Pistenma DA, Castellino RA, Meares EM. External beam radiation therapy of primary carcinoma of the prostate. Cancer. 1975;36(2):723-8.

4.Cupps RE, Utz DC, Fleming TR, Carson CC, Zincke H, Myers RP. Definitive radiation therapy for prostatic carcinoma: Mayo clinic experience. J Urol. 1980;124(6):855-9. 
5. Pilepich MV, Perez CA, Bauer W. Prognostic parameters in radiotherapeutic management of localized carcinoma of the prostate. J Urol. 1980;124(4):485-7.

6. Mahadevan A, Bucholz R, Gaya AM, Kresl J, Mantz C, Minnich DJ, Muacevic A, Medbery C $3^{\text {rd }}$, Yang J, Caglar HB, Davis JN. Best of the Radiosurgery Society ${ }^{\circledR}$ Scientific Meeting 2014: stereotactic radiosurgery/stereotactic body radiotherapy treatment of extracranial and intracranial lesions. Future Oncol. 2014;10(15):2307-10. doi: 10.2217/fon.14.168.

7. Hanks GE, Dunlap K. A comparison of the cost of various treatment methods for early cancer of the prostate. Int J Radiat Oncol Biol Phys. 1986;12(10):1879-81.

8. Perez CA, Michalski J, Ballard S, Drzymala R, Kobeissi BJ, Lockett MA, Wasserman TH. Cost benefit of emerging technology in localized carcinoma of the prostate. Int J Radiat Oncol Biol Phys. 1997;39(4):875-83.

9. Yu JB, Soulos PR, Herrin J, Cramer LD, Potosky AL, Roberts KB, Gross CP. Proton versus intensity-modulated radiotherapy for pros- tate cancer: patterns of care and early toxicity. J Natl Cancer Inst. 2013;105(1):25-32. doi: 10.1093/jnci/djs463.

10. Hoskin PJ, Rojas AM, Bownes PJ, Lowe GJ, Ostler PJ, Bryant L. Randomised trial of external beam radiotherapy alone or combined with high-dose-rate brachytherapy boost for localised prostate cancer. Radiother Oncol. 2012;103(2):217-22. doi: 10.1016/j.radonc.2012.01.007.

11. Hoffman RM, Barry MJ, Stanford JL, Hamilton AS, Hunt WC, Collins MM. Health outcomes in older men with localized prostate cancer: results from the Prostate Cancer Outcomes Study. Am J Med. 2006;119(5):418-25.

12. Stattin $\mathrm{P}$, Holmberg $\mathrm{E}$, Johansson JE, Holmberg L, Adolfsson J, Hugosson J; National Prostate Cancer Register (NPCR) of Sweden. Outcomes in localized prostate cancer: National Prostate Cancer Register of Sweden follow-up study. J Natl Cancer Inst. 2010;102(13):950-8. doi: $10.1093 /$ jnci/djq154.

13. Fox C, Romeijn HE, Lynch B, Men C, Aleman DM, Dempsey JF. Comparative analysis of $60 \mathrm{Co}$ intensity-modulated radiation therapy. Phys Med Biol. 2008;53(12):3175-88. doi: 10.1088/0031-9155/53/12/007.

14. Peeters ST, Heemsbergen WD, Koper PC, van Putten WL, Slot A, Dielwart MF, Bonfrer JM, Incrocci L, Lebesque JV. Dose-response in radiotherapy for localized prostate cancer: results of the Dutch multicenter randomized phase III trial comparing 68 Gy of radiotherapy with 78 Gy. J Clin Oncol. 2006;24(13):1990-6.

15. Zhu Z, Zhang J, Liu Y, Chen M, Guo P, Li K. Efficacy and toxicity of external-beam radiation therapy for localised prostate cancer: a network meta-analysis. Br J Cancer. 2014;110(10):2396404. doi: 10.1038/bjc.2014.197.

16. Bian SX, Kuban DA, Levy LB, Oh J, Castle KO, Pugh TJ, Choi S, McGuire SE, Nguyen QN, Frank SJ, Nguyen PL, Lee AK, Hoffman KE. Addition of short-term androgen deprivation therapy to dose-escalated radiation therapy improves failure-free survival for select men with intermediate-risk prostate cancer. Ann Oncol. 2012;23(9):2346-52. doi: 10.1093/annonc/mds001.

\title{
The way for optimization of distant radiation therapy in prostate cancer
}

\author{
Balkanov A.S.
}

Background: The rationale for distant gamma therapy (DGT) in prostate cancer patients is the data of its effectiveness in combination with modern planner devices, as well as lower costs, compared to the use of a linear accelerator.

Aim: To access the impact of DGT based on a 3D planning software "Amphora" on the 5-year survival rate depending on the main predictors of treatment efficacy in prostate cancer patients.

Materials and methods: The study included 34 prostate cancer patients who received low-dose DGT (total focal dose of 62 to $70 \mathrm{Gr}$ ). Androgen deprivation was used in $73.5 \%$ of patients for 6 to 12 months.

Results: The patients with a baseline prostate-specific antigen level $<20 \mathrm{ng} / \mathrm{ml}$ had the highest 5 -year survival rate of $86 \%$. There was no significant difference in a 5-year survival that depending on a total focal dose ( $<64 \mathrm{Gr} ; \geq 64 \mathrm{Gr}$ ) of DGT if used in combination with androgen deprivation.

Conclusion: This data represents only the first step in establishment of the criteria to choose the type of DGT in prostate cancer patients. The optimization of a treatment strategy based on a thorough analysis of data, including that on comorbidities and their severity, would make the use of DGT more rational that will reduce costs of treatment.

Key words: prostate cancer, radiation therapy, 5-year survival, costs of radiation therapy, prostate-specific antigen, elderly, comorbidity.

doi: 10.18786/2072-0505-2015-41-46-51
Balkanov Andrey Sergeevich - MD, PhD, Head of Department of Radiology ${ }^{1}$

$\triangle$ 61/2-7 Shchepkina ul., Moscow, 129110, Russian Federation. Tel.: +7 (495) 6811907.

E-mail: andreybalkanov@yandex.ru
Moscow Regional Research and Clinical Institute (MONIKI); 61/2 Shchepkina ul., Moscow, 129110, Russian Federation 